FORMATION Formation emploi

Revue française de sciences sociales

144 | Octobre-Décembre

Le décrochage scolaire à l'aune des inégalités

territoriales: nouvelles perspectives?

\title{
L'animation régionale de la prise en charge du décrochage scolaire : une action publique en tension
}

Regional management of school dropout : public action in tension

Der Umgang mit dem Schulabbruch auf regionaler Ebene : eine öffentliche Maßnahme unter Druck

La coordinación regional de la atención del abandono escolar : una acción pública en tensión

François Burban et Yves Dutercq

C OpenEdition

Journals

Édition électronique

URL : http://journals.openedition.org/formationemploi/6497

DOI : 10.4000/formationemploi.6497

ISSN : 2107-0946

Éditeur

La Documentation française

Édition imprimée

Date de publication : 31 décembre 2018

Pagination : $35-54$

ISSN : 0759-6340

Référence électronique

François Burban et Yves Dutercq, «L'animation régionale de la prise en charge du décrochage scolaire : une action publique en tension ». Formation emploi [En ligne], 144 | Octobre-Décembre, mis en ligne le 31 décembre 2019, consulté le 06 janvier 2021. URL : http://journals.openedition.org/ formationemploi/6497 ; DOI : https://doi.org/10.4000/formationemploi.6497

(C) Tous droits réservés 


\title{
L'animation régionale de la prise en charge du décrochage scolaire : une action publique en tension
}

\author{
FrançoIs BuRBan \\ Enseignant-chercheur en sciences de l'éducation et sociologue au Centre de recherche en \\ éducation de Nantes, université de Nantes \\ YVES DUTERCQ \\ Enseignant-chercheur en sciences de l'éducation et sociologue au Centre de recherche en \\ éducation de Nantes, université de Nantes
}

Résumé

L'animation régionale de la prise en charge du décrochage scolaire : une action publique en tension

Le nouveau contexte de la politique française de lutte contre le décrochage scolaire a suscité de fortes évolutions, dont notre article traite deux aspects liés : l'inflexion des politiques nationales et de leur animation par les services déconcentrés de l'Éducation nationale ; les actions et outils mis en œuvre par les conseils régionaux en matière de lutte contre le décrochage, en particulier les Plateformes de suivi et d'appui aux décrocheurs. Ce faisant, nous nous interrogeons sur l'articulation entre politique éducative nationale et déclinaison de l'action publique sur les territoires, dont l'efficacité se heurte aux divergences à la fois dans les logiques des institutions et dans celles de leurs agents.

Mots clés : abandon des études, mesure jeune, approche locale, organisation du travail, région, politique de l'éducation

Abstract

Regional management of school dropout: public action in tension

The struggle against school dropout in the context of the new French policy has led to major changes, two of them are discussed in our article: the reorientation of national policies and their management by the decentralized services of the National ministry of Education; the measures and tools implemented by the regional councils in the struggle against dropping out, in particular the "monitoring and support platforms for dropouts". Thus, we question the articulation between national educational policy and the local implementation of the public action whose effectiveness conflicts both with the logic of the institutions as well as those of their agents. 
Keywords: drop out, youth employment scheme, local approach, work organisation, region, education policy

Journal of Economic Literature: I 28 ; I 38 ; R 58

Traduction : Auteurs

\section{Introduction}

Quelles relations peut-on établir entre, d'une part, les préconisations nationales en termes de prévention du décrochage scolaire, de remédiation et d'accompagnement des jeunes sortant sans diplôme du système scolaire et, d'autre part, leurs traductions successives au niveau des territoires? Autour de quelles reconfigurations de logiques préexistantes se structurent les partenariats interinstitutionnels à l'échelle des territoires ?

Une instruction interministérielle d'avril 2009 (ministère de l'Éducation nationale, 2009) établit le cadre des nouvelles orientations politiques sur la question du décrochage scolaire. Elle définit en outre le décrochage comme le processus qui conduit un jeune en formation initiale à se détacher du système de formation jusqu'à le quitter avant d'avoir obtenu un diplôme. Un décret de fin 2010 (République française, 2010) fixe le niveau de qualification minimum que tout élève ou apprenti doit atteindre (baccalauréat ou diplôme à finalité professionnelle nationalement certifié). Il introduit également une définition du décrochage comme concernant "tout jeune qui quitte un système de formation initiale, sans avoir le niveau de qualification minimum requis par la loi ". La circulaire interministérielle de 2011 (ministère de l'Éducation nationale, 2011) reprécise les modalités du dispositif territorial préconisé de Plateformes de suivi et d'appui aux décrocheurs (PSAD). Ce dispositif engage plus concrètement la dimension interinstitutionnelle recherchée aux différents niveaux d'action, à partir du constat établi d'un faible pilotage national et d'une importante hétérogénéité dans son interprétation.

Après des années de mise sous l'éteignoir, la lutte contre le décrochage est donc enfin présentée comme une priorité nationale dans les années 2009-2010. Cependant, ce n'est que progressivement que les modes et moyens d'action à privilégier sont explicités, notamment à la suite des nouvelles impulsions données après l'alternance politique de 2012. C'est par exemple le cas du rapport des Inspections générales de 2013 (Inspection générale de l'Éducation nationale, Inspection générale de l'administration de l'Éducation nationale et de la recherche, 2013) qui met l'accent à la fois sur la prévention et sur la nécessité de lutter contre les causes internes, pointant donc la responsabilité de l'École. Mais c'est surtout la promulgation de la loi du 5 mars 2014 (République française, 2014) qui marque un tournant en transférant aux régions la coordination des actions de remédiation, en particulier via les PSAD. 
Ces nouvelles circonstances ont modifié la donne et en même temps suscité de fortes évolutions, dont nous traiterons ici deux dimensions : d'un côté, l'inflexion des politiques nationales, dont l'animation est désormais conduite par les services académiques ; d'un autre côté, la mise en œuvre, par les conseils régionaux, d'actions et d'outils de lutte contre le décrochage scolaire, en particulier dans le cadre des PSAD. L'enquête que nous avons menée à l'échelle d'un territoire privilégie, de ce fait, deux institutions : l'Éducation nationale, à travers son administration déconcentrée, et les services d'un conseil régional ${ }^{1}$. L'Éducation nationale s'appuie sur l'antériorité de son action menée dans le cadre de la Mission générale d'insertion (MGI), tandis que la Région se trouve investie de nouvelles prérogatives, par le transfert récent de la responsabilité des PSAD.

Or les deux institutions fonctionnent chacune selon une logique et avec des objectifs spécifiques qui tiennent à leur champ de compétences : l'Éducation nationale, qui fait de la scolarité et du diplôme les préalables incontournables à la construction de tout parcours social et professionnel, déploie une action visant avant tout la rescolarisation ; la Région, en charge de la formation et de l'insertion professionnelles, est en phase de renouvellement de ses compétences et de réorientation de ses moyens d'action, privilégiant l'articulation formation-emploi dans une visée d'affirmation de sa légitimité dans l'action publique territoriale.

Dès lors, notre contribution s'interroge sur la conjonction entre politique éducative nationale et déclinaison de l'action publique sur les territoires. De ce point de vue, la lutte contre le décrochage scolaire, considérée comme une priorité politique au moment de l'enquête, apparaît à la fois comme un révélateur et un analyseur pertinent de la réception des politiques publiques et des efforts pour leur modernisation.

Dans un premier temps, nous traitons du renouvellement des politiques de lutte contre le décrochage scolaire, dont un objectif majeur est de gagner en efficacité en privilégiant une dimension territoriale au plus proche des situations. Dans un deuxième temps, nous analysons comment cet objectif se traduit sur le territoire enquêté et ce qu'il suppose en termes de réorganisation des services et de coordination des actions des différentes institutions concernées. Dans un troisième temps, nous montrons que ces évolutions conduisent à la consolidation des réseaux déjà existants, au développement de logiques partenariales, mais aussi à la persistance de logiques institutionnelles. Létude des transformations qui résultent de la mise en œuvre territoriale des orientations récentes des politiques éducatives permet d'appréhender la finalité des actions et leur adéquation avec les attentes d'optimisation de l'action publique.

1. L'article est issu d'une recherche en cours menée dans le cadre du programme de l'Agence nationale de la recherche (ANR) Territoires et décrochage scolaire (TEDS) - convention ANR-14-CE30-0009. 


\section{Renouveler les politiques de lutte contre le décrochage scolaire pour gagner en efficacité ?}

Après avoir présenté les principaux concepts qui ont alimenté notre analyse (dépendance au sentier, logiques d'action, couplage et découplage de l'action collective, professionnalisme) et précisé notre méthodologie d'enquête (entretiens longs avec des acteurs nationaux et locaux de la lutte contre le décrochage), nous insistons sur l'intérêt d'une approche territoriale, en lien avec les nouvelles caractéristiques de la politique publique de lutte contre le décrochage scolaire.

\subsection{Un cadre conceptuel et des terrains d'investigation adaptés à une recherche multiniveau}

La lutte contre le décrochage scolaire suppose que les agents en charge de cette lutte, directement ou indirectement, renouvellent constamment leurs manières de faire et apprennent notamment à travailler avec des partenaires de plus en plus divers, au risque de se trouver en défaut de compétences, comme ils l'admettent eux-mêmes. Or, le recours à de nouveaux outils et à de nouvelles pratiques, ainsi que la coopération interinstitutionnelle et le travail en réseau constituent, dans le discours officiel, les gages d'une meilleure efficacité de la lutte contre le décrochage. Pour s'en persuader, il suffit de parcourir l'ensemble des préconisations des responsables politiques nationaux depuis 2009 et le plan Agir pour la jeunesse, inspiré du rapport de Martin Hirsch (HautCommissaire à la jeunesse, 2009).-

Toute organisation est traversée par des logiques d'acteurs contradictoires ou divergentes. Elle devient ainsi le lieu de compromis et de transactions entre ces diverses logiques (à dimension à la fois économique et sociale) soutenues par divers collectifs, internes et externes, ainsi que Boltanski \& Thévenot (1991) l'ont théorisé de façon générale. Or la recherche de partenariats interinstitutionnels est précisément le théâtre d'une confrontation des principes et des logiques d'action. En effet, ces partenariats ne sont pas de simples collaborations entre acteurs, mais des collaborations entre représentants d'institutions aux traditions fortes et aux cultures exacerbées, notamment en période de crise, comme c'est le cas pour l'institution scolaire, face à la dénonciation de l'incapacité de ses acteurs d'accomplir de façon satisfaisante leurs missions essentielles (la réussite des élèves, la lutte contre les inégalités, etc.). Les tensions qui brident le développement de partenariats tiennent à des divergences dans la définition de l'intérêt général, mais aussi à des enjeux de pouvoir qui s'expriment par des différences de pratiques préjudiciables à l'efficience de l'action publique.

C'est principalement par le phénomène de dépendance au sentier qu'on peut expliquer que les agents d'une organisation, tout spécialement d'une organisation fortement constituée autour de principes d'action éprouvés et culturellement ancrés, sont conduits 
à refuser de modifier leurs manières de faire dans des situations qui pourtant l'exigeraient (Palier, 2010). La notion de dépendance au sentier repose en effet sur l'idée que, même s'il existe une solution au problème posé plus efficace que la solution en cours, elle ne sera pas forcément retenue car elle se heurte à la force des habitudes et au coût, pour les acteurs, de procéder au changement (Maroy, 2000). North (1990) explique en particulier que la dépendance au sentier tient davantage au comportement des agents des institutions qu'aux technologies ou aux outils sur lesquels ils appuient leurs pratiques et qui pourraient en freiner matériellement l'évolution ou le changement. Plutôt que de s'engager dans de nouvelles voies, inconnues bien qu'éventuellement prometteuses, les agents refusent ou évitent de participer à l'évolution des institutions. Ils préfèrent conforter leur stabilité, pour ne pas avoir à faire face aux nécessités d'investissement, de formation, de coordination avec de nouveaux partenaires. Ce comportement a été largement observé dans des organisations bureaucratisées, comme peut l'être celle de l'éducation publique ou encore au sein de corporations aux puissantes traditions, comme celle des enseignants (Becker, 1951 ; Elliott, 1991 ; Dubet, 2002), dans la mesure où le changement s'y heurte à l'ancienneté, la permanence et la robustesse des règles et principes d'action.

Mais on peut tout autant supposer que des segments émergents dans des professions en mutation, tels que les coordonnateurs des Missions de lutte contre le décrochage scolaire (MLDS), s'agrippent à des modalités ou à des règles de fonctionnement nouvellement mises en place, qui leur servent de repères et de certitudes dans un monde où leur reconnaissance n'est pas encore acquise : ces " nouveaux professionnels » de la lutte contre le décrochage fonctionneraient alors à l'aune de la dépendance au sentier.

Or la politique d'efficience promue par le programme gouvernemental de Modernisation de l'action publique (MAP) se structure autour d'un ensemble d'instruments articulés les uns aux autres, dont en particulier le mode d'organisation des PSAD qui prend appui sur le partenariat interinstitutionnel. Ce mode d'organisation vise à décloisonner des politiques menées jusque-là de manière très sectorielle, tout spécialement quand il s'agit de l'action éducative, comme l'a montré Pons (2010).

Mais en matière de lutte contre le décrochage, Bernard \& Michaut (2014) font valoir que le partenariat interinstitutionnel peut se construire au nom de différentes logiques qui renvoient à autant de configurations du partenariat. Une première configuration, dans une logique administrative, cherche la réparation. Elle est portée par les agents de l'Éducation nationale, dont l'objectif est de rescolariser, de qualifier et de diplômer les décrocheurs et d'ainsi réparer ce que l'École a dans un premier temps échoué à réaliser. Une seconde configuration, dans une logique territoriale, vise la prévention de proximité. Il s'agit ici d'éviter le décrochage en détectant et accompagnant au plus près les élèves les plus exposés ou les plus vulnérables. Une troisième configuration, dans une logique de coéducation, privilégie la prévention organisationnelle, voire structurelle, 
qui intervient plus en amont encore, en particulier grâce à la prise de conscience et au travail des familles.

Nous montrons par la suite que le discours et l'action des hauts responsables de l'éducation interrogés cherchent non à créer des partenariats, qui existent déjà plus ou moins, même s'ils fonctionnent mal, mais à les faire basculer d'une logique administrative de réparation à une logique territoriale de prévention, la troisième configuration, la plus intersectorielle, n'étant encore en France qu'un horizon plus lointain, essentiellement tenu par les chercheurs et le monde associatif, par référence au travail mené par exemple au Canada depuis des années.

De façon générale, les partenaires potentiels de la lutte contre le décrochage éprouvent des difficultés à coordonner leur action. En effet, soit les territoires et compétences de chacun ne sont pas clairs, soit ces territoires et compétences font l'objet de fortes revendications et de forts clivages. En particulier, la mise en place des PSAD, qui suppose une étroite coordination entre institutions, tout spécialement l'Éducation nationale et la Région, au centre de la présente recherche, met en évidence les divergences d'interprétation et de niveau d'adhésion de leurs personnels respectifs en charge de la lutte contre le décrochage.

Les avancées récentes de la sociologie des professions, sous l'influence de l'approche néoinstitutionnelle ${ }^{2}$, ont mis en avant les notions de couplage et de découplage pour traiter de ces difficultés d'articulation qui nuisent à l'efficacité de l'action collective et génèrent des tensions en rapport avec l'aspiration au professionnalisme des partenaires (Freidson, 2001). Ces difficultés apparaissent même entre partenaires de l'action publique, dont chacun a une compétence qui correspond à son poste, comme on le voit au niveau des régions : dans notre cas, on citera l'Éducation nationale (mais aussi l'enseignement agricole), la formation professionnelle, la protection judiciaire de la jeunesse, etc.

Nous nous efforcerons à la suite de mettre en évidence les difficultés d'articulation entre le niveau national du pilotage de la politique de lutte contre le décrochage et le niveau de la mise en œuvre des mesures et dispositifs préconisés : le renouvellement des politiques nationales de lutte contre le décrochage scolaire passe par une conception qui privilégie la prévention, la coopération, l'autonomie de mise en œuvre et la mesure de l'efficacité. Autant de conditions qui se heurtent à des problèmes d'ordre technique, culturel et professionnel qui brident et contraignent les pratiques des acteurs locaux.

2. Le néo-institutionnalisme insiste notamment sur l'importance du contexte institutionnel, dont les règles déterminent fortement les acteurs et les phénomènes sociaux (voir par exemple Meyer \& Scott, 1983). 


\section{Encadré 1. Méthodologie}

Notre enquête se compose d'entretiens réalisés auprès d'acteurs des différents niveaux d'action (national, régional, local) et dans différentes sphères (Éducation nationale, formation professionnelle, protection de la jeunesse, associations et syndicats professionnels, Conseil régional). La présente contribution se réfère exclusivement à la première partie de cette enquête, menée aux deux bouts de la chaine. D'une part, avec des responsables nationaux de l'Éducation nationale ; d'autre part, avec des acteurs locaux de la lutte contre le décrochage d'un territoire correspondant à une région et une académie de l'Ouest de la France, exemplaires d'actions déjà engagées entre institutions, mais en aucun cas, comme nous le verrons, représentatives de l'ensemble des territoires qui présentent chacun une configuration spécifique.

Au total, vingt-trois entretiens d'une durée approximative d'une heure trente ont été réalisés aux niveaux national et territorial : six entretiens avec des hauts responsables de l'Éducation nationale, prescripteurs ou impliqués dans la politique de lutte contre le décrochage scolaire (un inspecteur général de l'administration de l'Éducation nationale (IGAEN), deux inspecteurs généraux de l'Éducation nationale (IGEN), deux inspecteurs généraux de l'Éducation nationale primaire (IGEN primaire), un chef de département) ; dix-sept entretiens avec les responsables territoriaux impliqués dans le pilotage des actions et dispositifs de lutte contre le décrochage scolaire (cinq responsables de Plateformes de suivi et d'appui aux décrocheurs (PSAD), un responsable d'une Mission de lutte contre le décrochage scolaire (MLDS), un représentant de la Protection judiciaire de la jeunesse (PJJ), deux représentants ville, deux responsables Région, un responsable de la Direction régionale de l'alimentation, de l'agriculture et de la forêt (DRAAF), quatre inspecteurs de l'Éducation nationale chargés de l'information et de l'orientation (IENIO), un responsable de la Chambre des métiers et de l'artisanat (CMA). Nous nous référons exclusivement, pour notre analyse, aux propos tenus par nos interlocuteurs dans le cadre de ces entretiens, en respectant le vocabulaire indigène et parfois technique utilisé, qui nous paraît le plus à même de rendre compte de cultures spécifiques.

\section{2. Privilégier l'entrée territoriale pour mieux prendre en compte la diversité des situations}

Comme en atteste le rapport des Inspections générales de 2013 (op. cit., 2013), les responsables de l'Éducation nationale ont pris acte de la nécessité de développer la prévention dans la lutte contre le décrochage. En effet, si la remédiation reste nécessaire à leurs yeux, elle leur paraît coûteuse, aléatoire et signe, dans tous les cas, l'échec d'un système scolaire dont ils sont de hauts représentants.

Dans le cadre de la politique de Modernisation de l'action publique, un rapport d'évaluation de la politique de lutte contre le décrochage scolaire a été remis en novembre 2014 (Direction générale de l'Enseignement scolaire, Secrétariat général pour la modernisation de l'action publique, 2014). Il s'appuie notamment sur une enquête menée auprès de jeunes bénéficiant des dispositifs d'aide au retour en formation ou en emploi. Il en ressort plusieurs préconisations d'ordre général. Elles ont trait aux nécessités d'évaluation, notamment par l'appel à des organismes indépendants, mais rapportent aussi l'efficacité souhaitée à la limpidité des objectifs politiques, l'allègement de la réglemen- 
tation, la simplification des procédures et l'autonomie de mise en ouvre. La proximité recherchée comme gage d'une meilleure appréhension des problèmes n'a en effet de portée qu'associée à une autonomie d'action suffisante, ainsi que l'explique un des inspecteurs généraux de l'Éducation nationale (IGEN) interrogés : "La proximité permet la réactivité, il y a là quelque chose de très intéressant. Et l'autonomie en matière éducative, en matière de prévention du décrochage, là aussi, c'est de pouvoir donner aux responsables de l'école les moyens, la liberté d'aller très vite à la recherche d'une liberté qui est d'ordre social et d'ordre médical. » (IGEN 1)

Certes, ces préconisations s'intègrent au mouvement plus général de responsabilisation des acteurs des politiques publiques, y compris ceux du niveau le plus local, mais il faut aussi considérer que ces derniers revendiquent en écho un travail en autonomie, gage sans doute de professionnalisme (Dutercq \& Maroy, 2017). Sur ce dernier point, le degré d'adéquation avec les préconisations des politiques éducatives nationales apparait plus significatif au niveau des managers des structures, dans et hors de l'Éducation nationale, qu'au niveau des opérateurs de terrain (Lipsky, 1980)

Après l'effervescence de 2001-2002, autant au niveau de la politique de la Ville qu'à celui de l'Éducation nationale, l'État a "oublié » la question du décrochage, incitant certaines collectivités territoriales à s'en emparer ou à s'en réemparer, comme ce fut le cas dans la région enquêtée. À partir de 2009, alors que l'État réinvestit ce champ, naît une période de tensions préjudiciables, d'une part, entre le gouvernement et l'Association des régions de France (ARF), d'autre part, entre certains recteurs et certaines régions, avec en arrière-plan des divergences idéologiques entre un gouvernement de droite et des conseils régionaux massivement de gauche, quatteste la délégation aux préfets de la tutelle des PSAD dans les années 2009-2013. Les régions ne sont ainsi que peu associées à la mise en place des PSAD, alors même que leurs prérogatives en matière de formation professionnelle semblaient appeler leur étroite collaboration, et en particulier la mobilisation des Centres de formation d'apprentis (CFA), dans une logique d'emploi évoquée plus haut : "Des régions davantage associées convaincraient davantage les CFA d'agir" (IGEN 3).

La volonté de meilleure coordination de l'action sur les territoires comme vecteur d'une plus grande efficacité s'est traduite, en 2014, par une mesure forte de régionalisation des PSAD et de l'orientation. La loi du 5 juillet 2014 stipule en effet que la région est "chargée de la politique régionale d'accès à l'apprentissage et à la formation professionnelle des jeunes et des adultes à la recherche d'un emploi ou d'une nouvelle orientation professionnelle ". Cela est un prélude à la transformation programmée du Service public d'orientation, créé en 2009, en Service public régional d'orientation (SPRO), qui avait été précédemment expérimentée dans la région concernée : "Au niveau régional, il n’y a pas que le transfert des plateformes, il y a aussi le transfert du service d'orientation et c'est là où il y a aussi des choses à rajouter dans une politique de prévention. Et le fait que plateformes et service public national d'orientation soient au même niveau, c'est quand 
même déjà une synergie qui peut sinstaller." (IGEN 2). "L'État a gardé la mainmise sur le système d'information mais, comme la loi le prévoyait, on va dans le sens d'une clarification de la demande, d'une meilleure explicitation des objectifs. On profite du nouveau découpage régional pour mieux se rapprocher des régions, de manière à traiter tous ensemble les problèmes, à faire émerger du collectif. C'est la clé de la réussite. "(DGESCO 1)

Pourtant, malgré le relatif consensus sur le caractère inéluctable de la régionalisation de l'orientation et de la formation professionnelle, incluant celle, désormais actée, de la prise en charge de la lutte contre le décrochage, sa mise en ouvre a d'emblée posé question.

Il y a d'abord risque de disparité interrégionale en matière de lutte contre le décrochage car, durant la période d'attentisme de l'État de la décennie 2000, certaines régions ont pris de l'avance, alors que d'autres, moins actives, se sont retrouvées en défaut d'expérience et de compétence. La lutte contre le décrochage a acquis, ces dernières années, une visibilité politique forte, attestée par sa mise aux agendas politiques régionaux. De plus, la mise en place d'une vraie politique régionale de lutte contre le décrochage nécessite un leadership assuré par le président de région ou par un/e vice-président/e. Or, rares sont, à ce niveau, les personnalités disposant d'une vision globale de la question. On trouve en effet, dans la plupart des cas au niveau régional, un/e responsable de l'éducation et un/e responsable de la formation professionnelle aux compétences distinctes et dont le couplage de l'action ne va pas de soi : les différences de culture, de logique institutionnelle, de leviers d'action et de sources de financement contribuent au contraire à susciter le découplage.

Par ailleurs, la décentralisation, entravée par des problèmes de statut et des questions d'ordre symbolique (légitimité institutionnelle, identités professionnelles) n'est pas achevée et crée des situations compliquées d'interférence entre prérogatives nationales et territoriales : il en est ainsi des Centres d'information et d'orientation (CIO), dont les personnels relèvent encore de l'Éducation nationale. Enfin, la régionalisation peut conduire à ce que l'action se réduise à la focalisation sur les risques externes, en minimisant la responsabilité à l'interne du système éducatif. Cette situation peut se trouver renforcée par le fait que les compétences des régions sont essentiellement de l'ordre de la coordination, dans la mesure où elles n'ont pas de réseau d'acteurs propre.

L'étude fine de la mise en place des PSAD sur un territoire nous permettra de mieux apprécier les difficultés d'ordre pratique et d'ordre symbolique auxquelles s'est heurtée la territorialisation de la politique de lutte contre le décrochage. 


\section{Encadré 2. Les acteurs territoriaux de la lutte contre le décrochage scolaire}

Le pilotage des actions de lutte contre le décrochage scolaire au niveau du territoire concerné est inscrit dans un partenariat ainsi que dans une coordination quadripartite qui comprend : le responsable de la Mission de lutte contre le décrochage scolaire (MLDS) (correspondant académique du ministère de l'Éducation nationale) ; un chargé de mission dédié à cette mission au sein du Conseil régional (représentant du président de Région) ; le préfet de Région ou un représentant délégué, ainsi qu'un représentant de la Direction régionale de l'agriculture de l'alimentation et de la forêt (DRAFF).

Les intervenants territoriaux dans la lutte contre le décrochage scolaire sont :

La MLDS, qui comprend, au niveau territorial, 31 pôles et un réseau de 56 coordonnateurs répartis en établissements sur toute l'académie. Elle agit essentiellement dans le domaine de l'intervention (29\%) et de la remédiation (71\%). Ses missions s'élargissent progressivement en amont, dans la prévention.

Les 16 Plateformes de suivi et d'appui aux décrocheurs (PSAD) de l'académie concernée, qui reposent sur une organisation partenariale. Elles sont placées sous la responsabilité du Conseil régional et animées par un responsable de l'Éducation nationale. C'est au niveau de ces PSAD que sont rassemblées les réponses possibles pour les jeunes décrocheurs, dont celles de l'Éducation nationale, par l'intermédiaire du réseau FOQUALE (Formation Qualification Emploi). À leur sortie des établissements, les jeunes sans diplômes peuvent contacter directement les PSAD ou sont signalés par leurs partenaires (Centres d'information et d'orientation, listes du Répertoire d'information et d'orientation, missions locales, Centres de formation d'apprentis (CFA), établissements d'enseignement publics et privés sous contrat, Pôle emploi, Missions insertion jeunes de l'enseignement catholique (MIJEC)).

\section{La prise en charge territoriale du décrochage scolaire ou le souci d'un travail de proximité}

L'hétérogénéité des modalités de coordination des actions de lutte contre le décrochage scolaire, d'un point de vue tant politique qu'opérationnel, est pointée dans un rapport de 2014 du programme de modernisation de l'action publique (Direction générale de l'enseignement scolaire, Secrétariat général pour la modernisation de l'action publique, op. cit., p. 2) : "Il faut dire que la mise en place nationale des PSAD par la circulaire de 2011 (ministère de l'Éducation nationale, op. cit.) a complexifié la coopération opérationnelle, notamment en raison de différences de niveau et de qualité de communication selon les institutions de rattachement, au premier rang desquelles les préfectures qui en ont la tutelle jusqu'en 2013. "(Responsable MLDS). La dimension interinstitutionnelle que cette circulaire cherchait à favoriser a conduit à des dysfonctionnements, en partie dus à des logiques administratives ancrées et prégnantes (Bernard \& Michaut, 2014). Cette approche descendante, fondée sur le contrôle et la régulation du dispositif, caractéristique du fort niveau de centralisation des politiques de lutte contre le décrochage scolaire en France (De Feudis, 2016), devait être compensée par un travail horizontal 
au niveau local, qui s'est révélé parfois difficile à réaliser en raison de la légitimité inégalement reconnue de l'animation des PSAD par les services de l'Éducation nationale.

Nous verrons ci-après en quoi les changements intervenus dans les orientations politiques ont une incidence sur les modalités organisationnelles des institutions en charge de les mettre en œuvre, tout en s'appuyant sur les coopérations et les partenariats historiquement constitués dans le territoire concerné. Ces transformations de structuration procèdent également d'une interprétation du sens des actions à mener pour lutter contre le décrochage scolaire et plus globalement pour articuler les actions interinstitutionnelles territoriales.

\subsection{De nouvelles modalités organisationnelles}

À la suite de la loi du 5 mars 2014, se met progressivement en place le transfert politique de la responsabilité des PSAD au président de Région. Le territoire sur lequel porte l'étude territoriale qui suit est jugé actif et même en avance par rapport à ce qui se fait au niveau national : la prise en compte du décrochage scolaire est déjà mise aux agendas et travaillée par les différents acteurs concernés depuis plusieurs années, avec cependant des degrés d'implication variables (Bernard \& Michaut, 2012). Un comité de pilotage intégrant les représentants des 16 plateformes, elles-mêmes constituées des acteurs des réseaux de formation initiale, des réseaux d'accueil et d'accompagnement des jeunes décrocheurs a été constitué autour du président de Région, du préfet et du recteur, afin de préparer la transition vers les PSAD.

Le conseil régional s'intéresse au décrochage, dont il n'a qu'une vision approximative. Afin de mieux appréhender ce dossier, son président désigne un cadre en charge de la coordination avec la MLDS, autour de deux rencontres hebdo madaires. Il commande également des études, notamment via le Conseil économique, social et environnemental (CESE). Il mobilise la cellule interne "innovation " du conseil régional, afin de faire des PSAD un outil opérationnel tenant compte de la dimension territoriale du dispositif : "Au $1^{e r}$ janvier de cette année [2015], la région a récupéré la coordination des fameuses PSAD, le président a dit: Moi, je souhaite effectivement récupérer cette compétence-là de la meilleure manière qui soit, en faisant en sorte de faire au moins aussi bien que ce que faisait l'État jusqu'à présent, et si possible, mieux. " (Directeur territorial, Conseil régional)

Le positionnement de la région sur le pilotage des plateformes est clairement présenté par les responsables du dossier du décrochage, au sein du conseil régional, comme s'inscrivant dans un design organisationnel ${ }^{3}$ (Pons, op. cit.) : "C'est une conviction très forte apportée par la DG. Il faut tout réinventer dans un contexte macro-économique et

3. On peut parler aussi de configuration organisationnelle, comme le fait Mintzberg (1989), ou d'agencement organisationnel. 
financier de plus en plus contraint qui impose de penser "global", et non plus de penser "en silo" ou de penser "expert", comme on pouvait le faire jusqu'à présent. " (Directeur territorial, Conseil régional)

Ce design organisationnel relève d'une possibilité d'extension et de mobilisation du réseau des partenaires institutionnels présents sur le territoire, notamment par rapport aux propositions de solutions apportées aux jeunes. Ce positionnement porte aussi sur les actions de prévention pouvant être mises en œuvre par les différents partenaires (Direction des lycées, de l'apprentissage, Direction emploi-formation) sous l'égide d'un directeur général délégué.

Le conseil régional cherche ainsi à orienter son action vers une logique d'action territoriale caractérisée par un travail en proximité avec les acteurs et les institutions travaillant déjà sur le décrochage. Cependant, l'organisation du dispositif repose sur une structuration antérieure, notamment liée aux lois de décentralisation. Elle s'inscrit dans une logique administrative de dépendance au sentier qui conforte l'organisation historiquement centralisée du conseil régional.

\subsection{Une approche globale facilitée par une organisation territoriale}

C'est ensuite à la demande du recteur que la Région confie l'animation des PSAD aux directeurs de $\mathrm{CIO}$. Cette décision est importante en termes de management des services publics car elle s'inscrit dans une continuité de l'organisation pratique préexistante, tout en marquant symboliquement une redistribution des autorités respectives. Au-delà des débats internes, et malgré la mise en place du SPRO, cette nouvelle répartition a été acceptée eu égard à la légitimité reconnue par le Conseil régional aux directeurs de $\mathrm{CIO}$ (70\% des jeunes venant de l'Éducation nationale) qui leur délègue la responsabilité des plateformes et l'organisation locale du partenariat : " $A$ l'origine, les préfets devaient mettre en ceuvre les PSAD, et ils se sont empressés de le passer au rectorat - parce qu'ils avaient l'expertise, etc. C'est une administration remarquable, avec des moyens et des personnels remarquables. Mais comme toute institution, à un moment donné, elle a du mal à sortir de ses références. " (Chef de service développement territorial - Conseil régional)

L'important apport financier du Fonds d'expérimentation pour la jeunesse ${ }^{4}$ (FEJ), obtenu par le rectorat lors de l'expérimentation du Livret de compétences expérimental (Haut-Commissariat à la Jeunesse/Fonds d'expérimentation pour la jeunesse, 20102012) auquel succédera le financement du Fonds social européen ${ }^{5}$ (FSE), permet de

\footnotetext{
4. Mis en place en 2009 par l'article 25 de la loi n²008-1249 du 1er décembre 2008, le Fonds d'expérimentation pour la jeunesse a pour objet de financer des programmes expérimentaux visant à favoriser la réussite scolaire et l'insertion sociale et professionnelle durable des jeunes.

5. Créé en 1957, le Fonds social européen est le principal levier financier de l'Union européenne pour la promotion de l'emploi, prioritairement en direction des groupes les moins qualifiés et les plus exposés au chômage et à l'exclusion.
} 
fédérer les services et les institutions autour de problématiques à traiter : "Depuis 2004, on n'a jamais eu autant de moyens. Chaque année, on a un peu plus de moyens, parce que, du coup, on a une grande maîtrise, maintenant, des fonds européens - qui donnent à ceux en qui ils ont confiance en fait " (Responsable MLDS). Cet appui sous-entend l'acceptation d'outils communs et partagés et le rendu de comptes dans les délais posés. L'ensemble cumulé de moyens humains et institutionnels, d'outils techniques (de repérage, analyse, traitement, communication...) et de pilotage, permet de structurer à la fois les services et l'action, s'inscrivant ainsi dans la ligne des préconisations du programme de modernisation de l'action publique (Direction générale de l'enseignement scolaire, Secrétariat général pour la modernisation de l'action publique, op. cit.).

L'histoire de la prise en charge du décrochage scolaire par les services académiques contribue à leur structuration progressive. Si l'expertise de l'académie est reconnue et qu'elle légitime sa position dans l'organisation fonctionnelle du dispositif, elle participe également à la structuration du partenariat interinstitutionnel par l'intégration du traitement de dossiers pouvant être mutualisés (accompagnement vers l'emploi ; allophonie ; handicap ; approches par compétences...) : "Les fonds européens sont un prétexte - c'est un prétexte idéal - à structurer, parce que si vous voulez plus de moyens, il va falloir faire ça un peu mieux que ça, il va falloir rendre compte, il va falloir prendre les outils, il faut respecter les délais. Voilà, donc, ça structure les choses. "(Responsable MLDS). Cette conception élargie du fonctionnement des services et du traitement transversal des dossiers entre les institutions contribue à développer une approche globale au niveau territorial. Cette approche cherche, d'une part, à intégrer les dimensions spatiales et temporelles spécifiques aux configurations territoriales ou locales et, d'autre part, à mutualiser les moyens et les ressources disponibles dans une logique de rationalisation et de pertinence portée par les politiques de modernisation de l’action publique.

\subsection{Logique de stocks ou logique de flux ?}

Conjointement au fonctionnement en proximité lors des comités techniques, le travail des opérateurs des PSAD est appuyé par le recours à une ressource numérique, le SIEI (Système interministériel d'échange d'informations), comme moyen organisé d'échange d'informations. Le SIEI "permet d'établir une liste finale de jeunes, sortis prématurément du cycle de formation initiale dans lequel ils étaient engagés, qui ne sont pas retrouvés dans un autre cursus de formation initiale et précise sills sont ou non accompagnés par une Mission locale ${ }^{6}$." (ministère de l'Éducation nationale, op. cit.). La constitution d'une base de données des jeunes concernés induit une coopération des établissements de formation initiale, des centres de formation des apprentis et des Missions locales.

6. Les finalités et le mode de fonctionnement de l'outil SIEI sont précisés dans le Bulletin officiel $\mathrm{n}^{\circ} 6 \mathrm{du} 10$ février 2011, «Lutte contre le décrochage scolaire, Organisation et mise en œuvre des articles L. 313-7 et L. 313-8 du Code de l'éducation ». 
Cependant, si les opérateurs reconnaissent un gain en termes de coordination des actions entre institutions, ils constatent que la qualité des données recueillies compromet la gestion des situations et par la suite, le partenariat institué : "Les listes créent beaucoup de désillusion. Parce que la liste, elle est très imparfaite, et à l'intérieur, en fait, les vrais décrocheurs sont à moins de $10 \%$. "(Responsable MLDS)

Ce problème est en partie lié à la temporalité de relevé des données et au fait que les listes constituées sont essentiellement limitées au repérage des jeunes. En effet, le SIEI prend en compte les données sur la base de deux indications annuelles, alors que les acteurs travaillent sur des relevés hebdomadaires, au plus proche des situations et de leurs évolutions, pouvant autoriser la mise en place d'actions de remédiation ou d'accompagnement. Ainsi, pour les opérateurs des PSAD, la tâche se déplace d'une " gestion de flux " vers une " gestion des stocks " (gestion et nettoyage des listes) jugée d'intérêt secondaire : "Le SIEI, c'est un outil qui est vraiment très critiqué par les animateurs. Il y en a qui le renseignent, et qui seraient susceptibles de l'exploiter sur le terrain... Et c'est toujours délicat de demander à des gens de faire quelque chose sans qu'ils en retirent un bénéfice. "(Inspecteur IO - Éducation nationale)

Les acteurs de terrain des différentes institutions demandent aux instances de pilotage le passage d'une logique de stocks à une logique de flux et donc une révision de l'organisation du travail des différents partenaires, eu égard au diagnostic posé et à la répartition des prises en charge par les PSAD : cette demande correspond à un recentrage de l'action des opérateurs sur la gestion concrète des cas, dimension plus en adéquation avec leurs attentes et leur " cœur de métier » qu’une gestion principalement administrative.

Si la fonction préventive de repérage est reconnue au SIEI, la légitimité des PSAD est fondée sur la capacité à mettre en place des actions d'accompagnement et de remédiation " au plus près " des situations singulières des jeunes. En ce sens, le pilotage des PSAD par les directions des CIO, qui articule prévention, accompagnement et remédiation, conforte les services académiques et constitue un enjeu stratégique pour, mais aussi au-delà de l'organisation territoriale de prise en charge de la question du décrochage scolaire.

\section{Vers une consolidation des réseaux territoriaux autour de logiques partenariales}

L'action territoriale engagée en faveur de la lutte contre le décrochage scolaire évolue globalement dans le sens des récentes orientations de la politique nationale, notamment en raison de la structuration historiquement constituée des actions respectives et partiellement concertée des institutions en présence. Toutefois, des logiques institutionnelles, relevant de dimensions culturelles, politiques ou économiques, perdurent 
et freinent en partie l'efficience recherchée. Un accord partiel se constitue alors à l'articulation d'enjeux pragmatiques - l'intérêt du jeune - et de la recherche d'une optimisation de l'action publique

\subsection{Persistance des logiques institutionnelles}

Le territoire enquêté est très diversifié dans sa constitution spatiale et démographique, ce qui laisse supposer des modalités de fonctionnement différentes, notamment pour la fréquence des rencontres : "Ce qu'on peut voir, c'est que les territoires de plateformes, vous avez quand même des grandes disparités en termes d'activité. Le problème, c'est que quand vous mettez en place un dispositif, il faut que vous ayez suffisamment de matière à traiter. L'outil, il est adapté à un flux. Quand vous avez de trop grandes disparités de flux, ça ne marchera pas pour les grandes... enfin, les plus grandes et les plus petites villes." (Chef de service développement territorial - (Conseil régional)

Au-delà de la dimension démographique, les disparités tiennent pour partie au fonctionnement local des institutions, qui peut conduire à ce que les flux apportés ne correspondent pas de facto à la configuration territoriale (taux et types d'équipements en organismes de formation et en établissements scolaires ; bassin d'insertion professionnelle, etc.). Des logiques d'appartenance et d'intérêt institutionnels interfèrent en effet dans la mise en commun des situations, comme dans le cas d'une des plateformes du territoire. Certains partenaires préfèrent encore conserver leur "vivier » de jeunes et les solutions qu'ils sont à même de proposer plutôt que soumettre les cas qui pourraient trouver une issue extérieure aux réseaux de leur institution : "Il y a une vraie difficulté là-dessus, c'est que les autres sortes de solutions qui existent - et il y en a : formation continue, apprentissage, service civique, emploi - sont finalement peu mobilisées. " (Direction régionale de l'alimentation, de l'agriculture et de la forêt)

Une des finalités recherchées par la mise en place des PSAD autour d'une action concertée des différentes institutions est d'infléchir des logiques de fonctionnement préexistant dans les divers canaux institutionnels. Cependant, l'hétérogénéité dans l'interprétation de la commande et la constitution des listes relèvent d'intérêts différents pour les institutions concernées ; en effet, la création plus ou moins artificielle d'un public de décrocheurs, par une interprétation extensive de la population relevant des dispositifs mis en place, peut permettre d'accéder à des financements dédiés. Ces conflits d'intérêt engendrent des tensions entre les différentes institutions et peuvent mettre à mal le partenariat en cours de constitution. Leur positionnement dans le débat sur les questions de stock et de flux révèle des logiques d'action qui appréhendent diversement les possibilités de traitement des situations des jeunes.

Les tensions et la concurrence qui en découlent peuvent être liées au financement des missions qui dépend de canaux institutionnels distincts, au point que certains interlocuteurs parlent de l'émergence d'un " quasi-marché » de la gestion des publics en 
difficulté ou d'une véritable " guerre des pauvres ${ }^{7}$ (selon l'expression d'un responsable MLDS : l'accompagnement de publics en difficulté permet à différents services d'obtenir leurs financements, en fonction des résultats de leurs actions. Cette politique du chiffre participe également à leur reconnaissance et à la médiatisation de leur action, véritable enjeu politique pour les institutions.

\subsection{Finalité des actions et optimisation de l'action publique}

La réflexion conduite autour du fonctionnement des PSAD et, plus globalement, du pilotage et de la concertation des actions des différentes institutions repose sur la recherche de l'efficience aux différents niveaux. Ainsi, deux objectifs déterminants aboutissent à un consensus et à la structuration de l'action concertée des services interinstitutionnels.

D’une part, d'un point de vue pragmatique, il s'agit de trouver la réponse la plus adéquate à la problématique singulière de chaque jeune en situation de décrochage. Pour cela, il est nécessaire de mettre à plat les solutions possibles (retour à la scolarité, entrée en formation, accompagnement à l'insertion professionnelle, etc.), ce qui suppose une concertation des institutions, donc une rupture au moins partielle avec les dépendances au sentier existantes. D'autre part, d'un point de vue politique et économique, il faut rechercher une optimisation de l'action publique. Cet objectif suppose une rupture avec les logiques concurrentielles précédemment décrites, par une concertation des actions et une optimisation des moyens qui lui sont alloués.

La présentation des PSAD comme relevant d'un intérêt complémentaire, à la fois pour les bénéficiaires et pour les politiques publiques, est revendiquée par le responsable de la MLDS : "J'ai toujours défendu ça auprès de mes supérieurs et puis de la région et autres, c'est que c'est une optimisation des politiques publiques... Je propose au recteur que la MLDS ne soit pas uniquement l'instrument d'Éducation nationale pour Éducation nationale." (Responsable MLDS)

Il faut cependant parallèlement insister sur l'orientation privilégiée par les responsables académiques rencontrés (MLDS, inspecteur information et orientation, CIO), en écho au discours des hauts responsables de l'Éducation nationale, vers les actions de prévention plutôt que vers celles de " traitement " ou de "remédiation ». Au-delà de sa dimension économique ou morale, le choix de la prévention peut être aussi un choix d'intérêt, puisque les actions de prévention portent sur des jeunes qui sont encore d'âge scolaire. On peut enfin considérer que ces divergences renvoient à une division du travail entre les institutions, la remédiation concernant plus particulièrement celles dont les actions se situent en aval du système scolaire et sont plus directement orientées vers l'insertion professionnelle. Dans ce sens, si cette structuration est plus proche de la logique territoriale attendue de toutes parts, elle n'en constitue pas moins une forme de continuité des

7. Nous renvoyons sur ce point à l'article de J. Zaffran \& J. Vollet, dans Formation Emploi, 2018, nº 143. 
modalités antérieures de fonctionnement et des logiques administratives ou de dépendance au sentier qui les accompagnent.

La conjonction des actions engagées au niveau du territoire et des orientations retenues par les différentes hiérarchies institutionnelles, en accord avec les récentes décisions politiques nationales, débouche sur un consensus territorial sur le fonctionnement et le pilotage du dispositif PSAD. Malgré les résistances, il ressort qu'une dynamique est enclenchée. En ce sens, il est possible d'avancer l'hypothèse qu'une logique territoriale se développe parallèlement ou en suppléance partielle à la logique administrative dominante dans le système français. Notons que cette évolution est cependant fondée en légitimité dans une logique administrative inscrite dans les préconisations récentes du programme de modernisation de l'action publique (Direction générale de l'enseignement scolaire, Secrétariat général pour la modernisation de l'action publique, op. cit.).

Soulignons enfin que la majorité des représentants institutionnels rencontrés s'accorde à dire que la dynamique initiée en 2014 par le transfert des PSAD est largement tributaire de l'existant au niveau régional ${ }^{8}$, au point que, sur certains territoires, le travail se limiterait principalement au traitement des listes de cas identifiés par le SIEI (stock). Pour nos interlocuteurs, l'engagement a minima des territoires dans le processus de décentralisation, lié à la mise en place des PSAD, risque de condamner leur activité. La dynamique insuffisante sur certains territoires s'expliquerait en partie par l'insuffisante dotation en personnel dédié au fonctionnement des PSAD.

\section{Conclusion}

Il ressort d'abord de notre étude de la territorialisation de l'action publique de lutte contre le décrochage scolaire que la différence des niveaux d'action se traduit par des déclinaisons politiques, des préconisations et un outillage parfois éloignés des situations de terrain (Bernard \& Michaut, 2012). Ainsi, les difficultés posées par la mesure et l'évaluation de la pertinence des actions à l'échelle du local et de petits espaces justifient la nécessité clamée par les acteurs locaux de travailler au plus près des situations caractérisées par leur grande diversité.

Par ailleurs, le constat de disparités territoriales flagrantes en matière de décrochage aide à mieux comprendre que, malgré les doutes liés aux difficultés du partenariat interinstitutionnel, l'entrée territoriale a été privilégiée au long de ces dernières années, tant elle paraît la plus à même de prendre à revers les traditions inhibantes et les résistances au changement (North, 1990 ; Palier, 2010). Mais c'est en même temps bien là qu'on perçoit la persistance de la tension entre logique administrative visant la réparation et logique territoriale privilégiant la prévention.

8. Nous renvoyons sur ce point à l'article de T. Berthet, A. Brizio et V. Simon dans ce même dossier. 
Pourtant, l'antériorité du travail réalisé au niveau de notre territoire d'enquête par rapport à d'autres (disparité interrégionale), couplée à la réflexion menée depuis des années par les partenaires de la lutte contre le décrochage scolaire, rend plus aisées les évolutions des modalités de travail qu'appelle la concertation politique au niveau régional. L'action conduite par les responsables territoriaux des différentes institutions tend en effet à une mise en cohérence horizontale et verticale. Elle est en outre argumentée sur la base d'un accord autour d'un objectif commun, l'intérêt du jeune.

La priorité accordée à la prise en compte du décrochage aboutit à une entente sur une problématique d'intérêt général, mais se heurte aussi à des déclinaisons hétérogènes des institutions (Éducation nationale, Région, etc.) (Bernard \& Michaut, 2012). Ce processus est donc fragile, en raison notamment du transfert de la responsabilité des PSAD aux régions, mais également des dépendances au sentier, des divergences dans les intérêts « économiques » et dans le sens et les finalités de l'action entreprise.

Impossible cependant de nier qu'une réelle dynamique d'efficacité dans la prise en charge du décrochage s'est enclenchée. Deux hypothèses explicatives peuvent être à ce stade avancées : soit la logique territoriale se substitue progressivement à la logique administrative qui caractérise les institutions françaises ; soit il n'y a pas, au bout du compte, contradiction fondamentale entre les logiques et entre les revendications portées par les différents acteurs de la lutte contre le décrochage. Cela conduit, bon an mal an, au renforcement de l'action collective, à la coopération ou, plus précisément encore, au couplage entre l'action de partenaires aux spécificités, aux cultures et aux professionnalismes identifiés.

Ces deux hypothèses se rejoignent sur l'idée d'un consensus vers lequel doivent tendre les négociations et les régulations réalisées au niveau territorial. En outre, elles recoupent, au moins partiellement, dans une situation et pour un temps donnés, les récentes préconisations nationales des politiques éducatives en faveur d'une harmonisation des différents niveaux d'action (Direction générale de l'enseignement scolaire, Secrétariat général pour la modernisation de l'action publique, 2014).

\section{Bibliographie}

Becker H. S. (1951), Role and career problems of the Chicago public school teacher, PhD Thesis, University of Chicago Press.

Bernard P.-Y. \& Michaut C. (2012), « Pourquoi expérimenter une politique éducative? Le repérage des jeunes en décrochage scolaire ", Revue française de pédagogie, 181, pp. 5-14.

Bernard P.-Y. \& Michaut C. (2014), « Le partenariat interinstitutionnel : un nouvel instrument de politique éducative ", Education comparée 11, pp. 111-131. 
Boltanski L. \& Thévenot L. (1991), De la justification, Paris, Gallimard.

De Feudis E. (2016), Les politiques régionales de lutte contre le décrochage scolaire en Italie, France et Espagne, Thèse de science politique en cours, Université la Cattolica de Milan.

Direction générale de l'enseignement scolaire, Secrétariat général pour la modernisation de l'action publique (2014), Evvaluation partenariale de la politique de lutte contre le décrochage scolaire plus efficace. Rapport MEN-SGMAP (novembre 2014). Paris, ministère de l'Éducation nationale.

Dubet F. (2002), Le Déclin de l'institution, Paris, Seuil.

Dutercq Y. \& Maroy C. (Eds.) (2017), Le travail et le professionnalisme enseignants face aux politiques de responsabilisation, Bruxelles, De Boeck.

Elliott J. (1991), Action Research for Educational Change, Buckingham, Open University Press.

Freidson E. (2001), Professionalism: The third logic, Chicago, University of Chicago Press.

Haut-Commissaire à la Jeunesse (2009), Livret vert. Commission sur la politique de la jeunesse, Paris : Haut-Commissaire à la jeunesse. <http://www.ladocumentationfrancaise.fr/var/storage/rapports-publics/094000308.pdf>

Inspection générale de l'Éducation nationale, Inspection générale de l'administration de l'Éducation nationale et de la Recherche (2013), Agir contre le décrochage scolaire: alliance éducative et approche pédagogique repensée. Rapport au Ministre de l'éducation nationale, Paris, ministère de l'Éducation nationale.

Lipsky M. (1980), Street-Level Bureaucracy: Dilemmas of the Individual in Public Services, NewYork, Russell Sage Foundation.

Meyer J. \& R. Scott (1983), Organizational Environments. Ritual and Rationality, Beverly Hills, Sage Publications.

Ministère de l'Éducation nationale (2009), Prévention du décrochage scolaire et accompagnement des jeunes sortant sans diplôme du système scolaire, Instruction $\mathrm{n}^{\circ} 09-060 \mathrm{JS} \mathrm{du}$ 22-4-2009, Bulletin officiel ${ }^{\circ} 23$ du 4 juin 2009.

Ministère de l'Éducation nationale (2011), "Circulaire n²011-028 du 9 février 2011, Organisation et mise en œuvre des articles L. 313-7 et L. 313-8 du code de l'Éducation ", Bulletin officiel n ${ }^{\circ} 6$ du 10 février 2011.

Minzberg H. (1999), Le management. Voyage au centre des organisations, Paris, Éditions d'organisation. 
North D. (1990), Institutions, Institutional Change and Economic Performance, Cambridge, Cambridge University Press.

Palier B. (2010), "Path dependence (Dépendance au chemin emprunté)", in Dictionnaire des politiques publiques, Paris, Presses de Sciences Po, pp. 411-419.

Pons X. (2010), Évaluer l'action éducative, Paris, PUF.

République française (2010), « Décret n 2010-1781 du 31 décembre 2010 fixant le niveau de qualification prévu à l'article L. 313-7 du code de l'Éducation ", JORF n $0001 \mathrm{du} 1$ janvier 201, texte $\mathrm{n}^{\circ} 39$.

République française (2014), "Loi $\mathrm{n}^{\circ}$ 2014-288 du 5 mars 2014 relative à la formation professionnelle, à l'emploi et à la démocratie sociale ", $O R F \mathrm{n}^{\circ} 0055$ du 6 mars, page 4848 , texte $n^{\circ} 1$. 